\title{
Síndrome ocular isquêmica secundária à arterite de Takayasu - Relato de caso
}

\author{
Ocular ischemic syndrome secondary to Takayasu's arteritis - case report
}

\author{
Marcos Antonio Barbosa do Vale ${ }^{1}$ \\ Patrícia Brito ${ }^{2}$ \\ Marco Polo Ribeiro ${ }^{3}$ \\ Maria Amélia Bulhões ${ }^{4}$
}

\begin{tabular}{|c|}
\hline RESUMO \\
\hline A síndrome ocular isquêmica representa manifestação da hipoperfusão \\
crônica do suprimento arterial ocular. A arterite de Takayasu caracteriza- \\
se pelo acometimento de grandes vasos, com graus variáveis de obstru- \\
ção, geralmente ramos da aorta. O objetivo deste trabalho é relatar um caso \\
de síndrome ocular isquêmica secundário à arterite de Takayasu. O exame \\
da angiografia fluoresceínica digital evidenciou presença de sinais de \\
hipoperfusão coriorretiniana e shunts arteriovenosos, e a arteriografia \\
mostrou sinais de diminuição acentuada do fluxo sangüíneo de ambas as \\
carótidas, direita e esquerda, acima de $90 \%$.
\end{tabular}

Descritores: Arterite de Takayasu/complicações; Isquemia/etiologia; Artéria retiniana/ oclusão; Angiofluoresceinografia/métodos; Síndrome; Retina/fisiopatologia; Artérias carótidas/radiografia; Nervo óptico/irrigação sangüínea; Relato de caso
Trabalho realizado no Hospital de Olhos Ruy Cunha (Day Hospital de Olhos Ruy Cunha), Salvador - BA. Coordenador do Departamento de Retina e Vítreo do Day Hospital de Olhos Ruy Cunha.

${ }^{2}$ Coordenadora do Departamento do Oftalmopediatria e Visão Sub Normal do Day Hospital de Olhos Ruy Cunha.

${ }^{3}$ Interno do 6⿳a ano do Hospital de Olhos Ruy Cunha Salvador, Acadêmico da Escola Bahiana de Medicina e Saúde Pública - Salvador - BA.

${ }^{4}$ Cardiologista da Fundação Bahiana de Cardiologia-Hospital Universitário Prof. Edgar Santos - Salvador - BA.

Endereço para correspondência:

E-mail: mbvale@uol.com.br

Recebido para publicação em 27.10.2000

Aceito para publicação em 20.08.2001

\section{INTRODUÇÃO}

A síndrome ocular isquêmica (SOI), descrita pela primeira vez por Brown, compreende a manifestação de hipoperfusão crônica do suprimento arterial ocular, que pode levar a comprometimento funcional da visão ${ }^{(1)}$. Geralmente é secundária à ateroesclerose das artérias carótidas, mas pode estar relacionada à arterite de Takayasu ou arterite de células gigantes, e mais raramente à estenose de artéria oftálmica ${ }^{(1-3)}$.

A arterite de Takayasu foi inicialmente demonstrada no Japão, em 1908 por Takayasu* que observou alterações oculares em mulheres jovens portadoras de vasculopatia carotídea (citado por Haimovici, 1989).

Em 1951, Shimizu e Shikano** descreveram clinicamente a doença, e depois, vários relatos de caso mostraram que acomete todas as raças em todas as partes do mundo (citado por Haimovici, 1989). Pode ser denominada como síndrome do arco aórtico, síndrome de Martorell, coarctação da aorta atípica, arterite braquiocefálica e aortite idiopática ${ }^{(4)}$.

É uma arterite obstrutiva inespecífica, de caráter imunológico, na qual são acometidas as artérias de grande calibre por processo inflamatório granulomatoso, geralmente ramos da aorta, que leva a uma obstrução secundária à esclerose fibrosante ${ }^{(4-5)}$.

\footnotetext{
* Takayasu M. Cases with unusual changes of the vessels in the retina. Acta Soc. Ophthalmology Japan, 1908;12:554. apud Haimovici H, Mishima Y. Non atherosclerotic diseases of small arteries. In: Haimovici H. Vascular surgery: principles and techniques. 3th.ed. Norwalk, Conn: Appleton \& Lange; 1989. p.432-5

** Shimizu K, Shikano K. Pulseless disease. J Neuroophthalmol Exp Neurol, 1951;1:37. apud Haimovici H, Mishima Y. Non atherosclerotic diseases of small arteries. In: Haimovici H. Vascular surgery: principles and techniques. 3th.ed. Norwalk, Conn: Appleton \& Lange; 1989. p.432-5
} 
O objetivo deste trabalho é relatar um caso de síndrome ocular isquêmica secundária a arterite de Takayasu discutindo seus achados clínicos.

\section{RELATO DE CASO}

M. F. S., 46 anos, do sexo feminino, queixa-se de baixa acuidade visual há um ano e meio. Antecedente de facectomia com implante de lente intra-ocular no olho direito em maio de 1999 e olho esquerdo em julho de 1999. Em uso de fluormetalona três vezes ao dia. Ao exame, a acuidade visual corrigida do olho direito e do olho esquerdo era de conta-dedos a meio metro, a pressão intra-ocular, em ambos os olhos de $10 \mathrm{mmHg}$ às 11:50h. No exame biomicroscópico observou-se, em ambos os olhos, córnea transparente, câmara anterior média, sem células ou "flare", ausência de neovascularização de íris ou de ângulo e pseudofacia.

O exame da retina demonstrava uma rarefação difusa do epitélio pigmentar da retina, hemorragias intrarretinianas em pólo posterior; ingurgitamento venoso e presença de obliteração vascular extensa (Figura 1A e 1B).

A angiografia fluoresceínica digital apresentou retardo do tempo braço coróide, cerca de 25 segundos. Observou-se presença de "shunts" arteriovenosos no pólo posterior do fundo de olho de ambos os olhos, com grande extravasamento nas fases tardias e denotando edema macular. A periferia mostrou sinais de baixa perfusão (Figura 2A e 2B).

$\mathrm{O}$ eletrorretinograma (ERG) demostrou diminuição da amplitude das respostas em todas as fases do exame sugerindo perda funcional significativa da atividade retiniana em todas as camadas. Na fase escotópica, a resposta máxima combinada, apresentou significativa diminuição da amplitude das ondas A e B, e aumento da latência da onda A de ambos os olhos (Tabela 1).

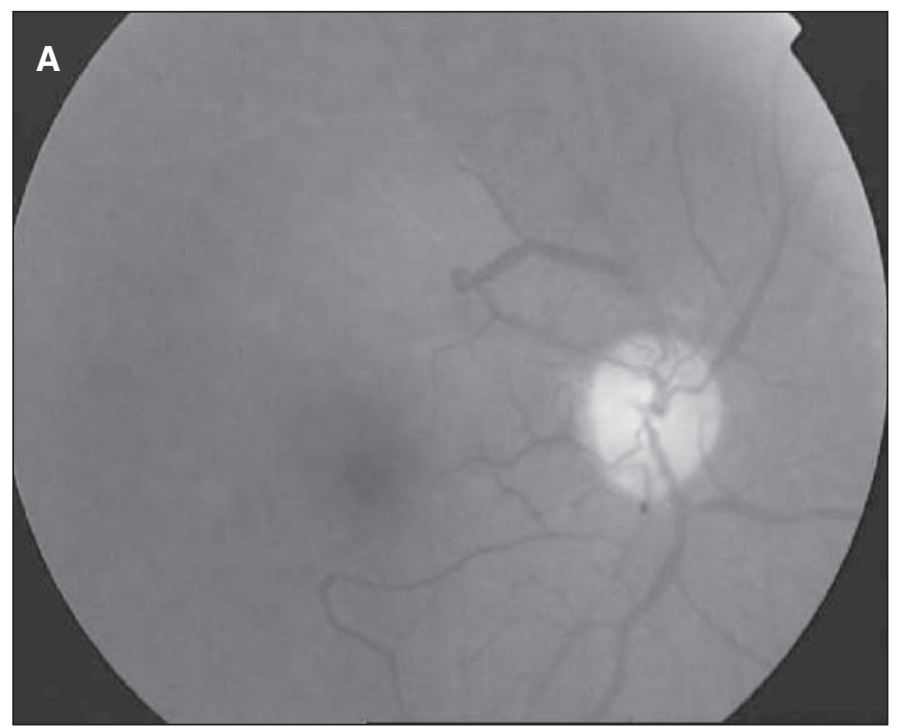

Figura 1 - A: Retinografia do fundo do olho direito; B: Retinografia do fundo do olho esquerdo

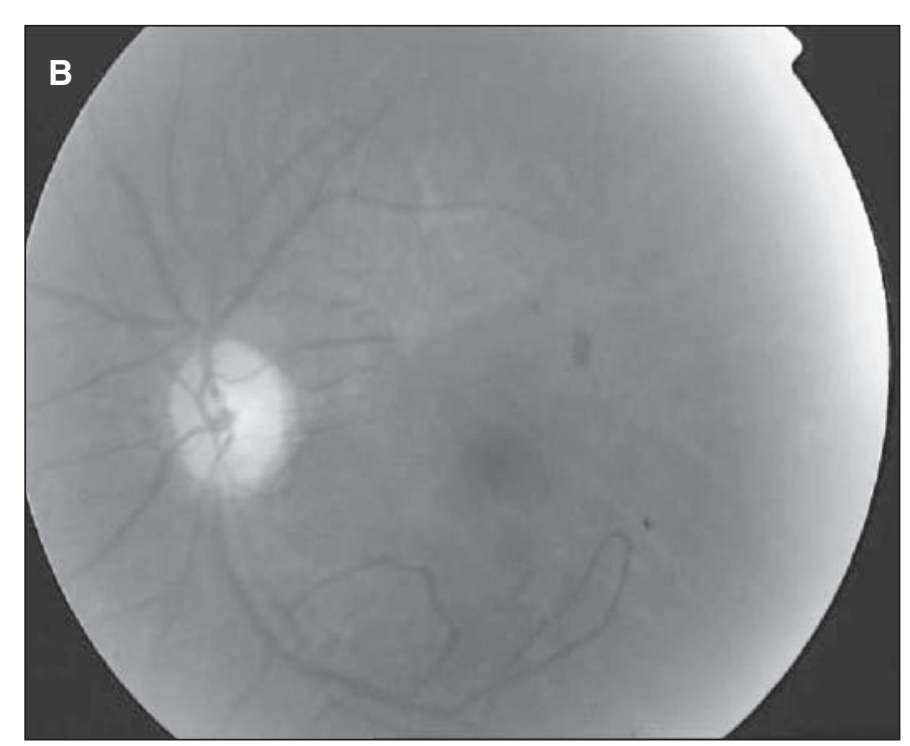

Diante dos sinais de sofrimento isquêmico ocular, sugerindo alterações vasculares, solicitamos extensa avaliação laboratorial incluindo hemograma completo, leucograma, glicemia de jejum, perfil lipídico, provas de função renal e hepática, sumário de urina, velocidade de hemossedimentação (VHS), antiestreptolisina- $\mathrm{O}$, proteína $\mathrm{C}$ reativa, fator antinúcleo, fator reumatóide, eletroforese de proteínas plasmáticas, perfil tireoidiano.

Para confirmar diagnóstico foi solicitado eletrocardiograma, ecodopplercardiograma, cintilografia tomográfica de perfusão miocárdica, ultra-sonografia com Doppler de carótidas e arteriografia. rol total $(291 \mathrm{mg} / \mathrm{dl}$ com LDL de $220 \mathrm{mg} / \mathrm{dl})$ e VHS (25 $\mathrm{mm} \mathrm{1}^{\text {a }}$. hora). Os demais exames laboratoriais encontravam-se dentro dos padrões da normalidade. O eletrocardiograma, o ecodopplercardiograma, a cintilografia tomográfica de perfusão miocárdica não mostraram alterações.

O ultra-som com Doppler de carótidas observou ausência de fluxo sangüíneo, nas carótidas esquerda e direita, sugerindo obstrução de ambas as carótidas comuns.

A arteriografia demonstrou oclusão da artéria subclávia direita na origem, artéria subclávia esquerda com estenose de 75 a $90 \%$. A artéria carótida comum direita apresentava estenose de $90 \%$ proximal, não sendo possível observação distal das artérias carótidas internas e externas. A artéria carótida esquerda estava ocluída na origem. Artéria vertebral esquerda com estenose ostial de 75\%. O exame demonstrou comprometimento importante da perfusão de ambas as carótidas, sendo os achados compatíveis com arterite de Takayasu (Figura 3).

\section{DISCUSSÃO}

A SOI caracteriza-se por uma hipoperfusão crônica do suprimento arterial ocular ${ }^{(1)}$. Nesses pacientes a incidência de
A avaliação laboratorial demonstrou aumento no coleste- 


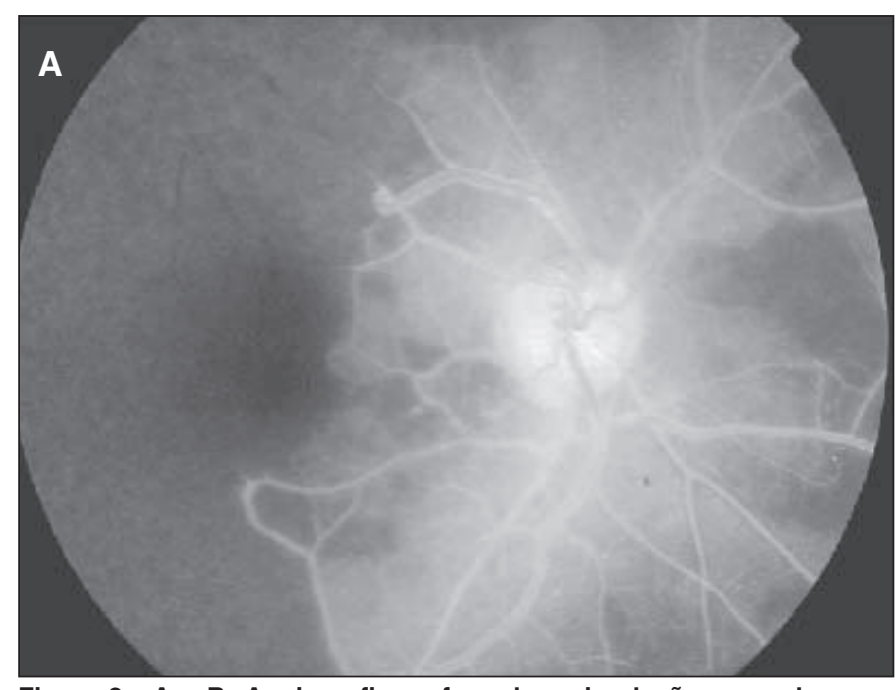

Figura 2 - A e B: Angiografia na fase de recirculação em ambos os olhos
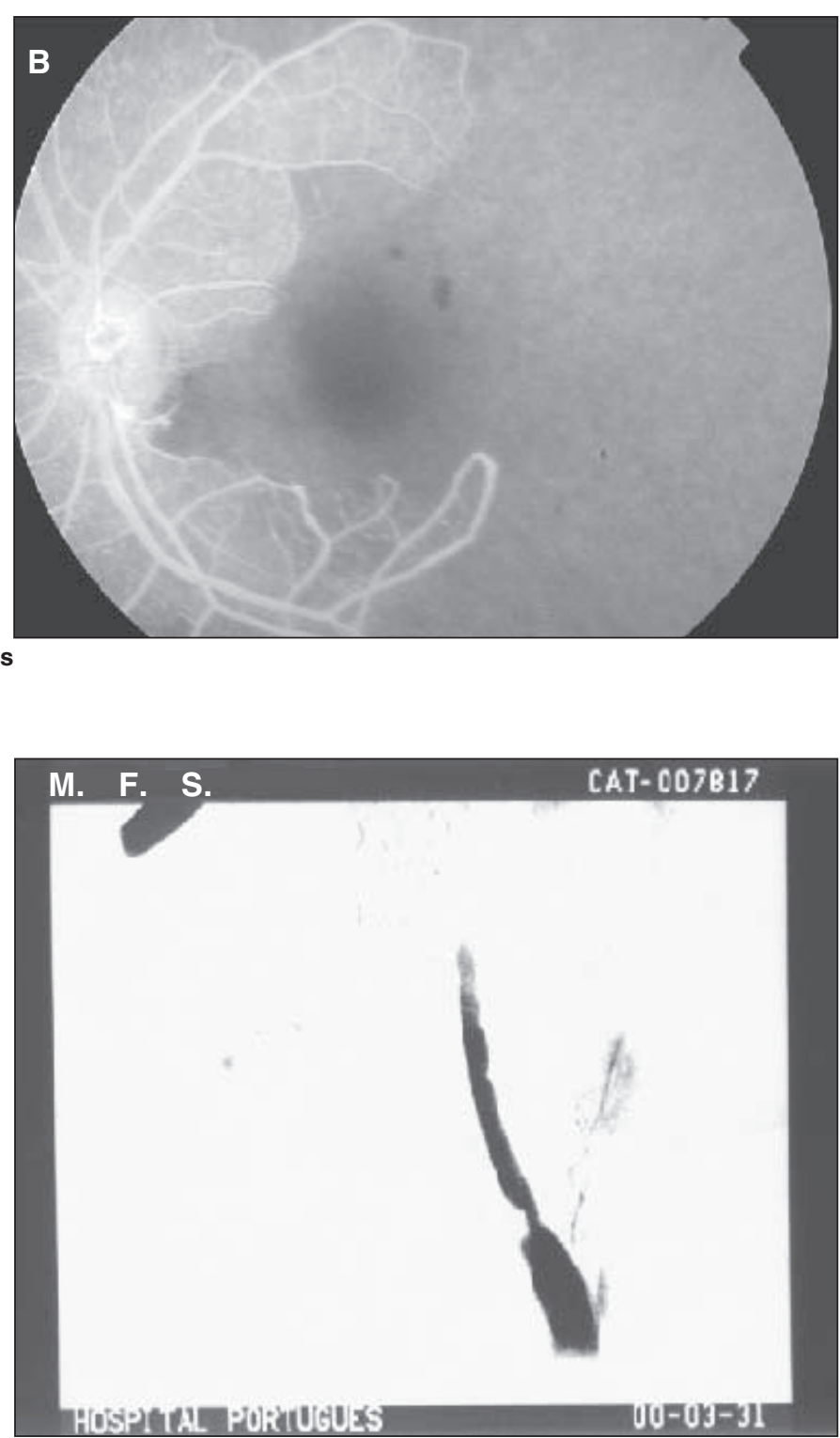

Figura 3 - Tronco braquiocefálico mostrando importante estenose

suas camadas. A onda A, representa resposta dos fotorreceptores, encontra-se diminuída pelo sofrimento isquêmico coroideano, e a onda B, que representa a resposta elétrica das camadas mais internas da retina, encontra-se diminuída e às vezes negativa devido ao comprometimento da perfusão retiniana ${ }^{(3)}$.

O exame ultra-sonográfico com Doppler da artéria carótida, apesar de não ser tão preciso quanto à angiografia da artéria da carótida, oferece bons resultados além de ser um exame não invasivo $^{(7)}$.

Nesse caso a SOI se apresentou secundária à arterite de Takayasu, que se caracteriza por acometer mulheres jovens, inicialmente com ataques de amaurose fugaz e síncope ${ }^{(4)}$. Não existem sinais ou sintomas clínicos patognomônicos, podemos encontrar uma variabilidade clínica, desde febre de origem desconhecida até sintomas neurológicos, os pacientes 
geralmente se queixam de inaptidão e claudicação de membros, dependendo dos ramos aórticos acometidos e de sua intensidade ${ }^{(4)}$. Nos resultados de exames laboratoriais podemos encontrar leucocitose, VHS elevada e proteína $\mathrm{C}$ reativa presente, numa incidência de 35 a 53\% dos $\operatorname{casos}^{(4)}$.

Os achados oculares mais marcantes são os "shunts" arteriovenosos demonstrados na angiografia fluoresceínica. Outros achados comuns são congestão de veias retinianas, extravasamento, neovascularização, microaneurismas, e menos comumente observam-se manchas algodonosas, NOIA e êmbolos retinianos ${ }^{(4,8-10)}$.

O tratamento da arterite de Takayasu consiste no uso de esteróides a longo prazo quando é diagnosticada na fase inicial, pode ser controlado pelos dados laboratoriais que indicam a sua atividade como VHS e proteína $\mathrm{C}$ reativa ${ }^{(4,11)}$. Quando as obstruções estão presentes, a terapia com esteróide não se mostra eficaz, pois não reverte as alterações anatômicas e funcionais. A indicação cirúrgica é feita em cerca de $9 \%$ dos casos e limita-se à isquemia cerebral e claudicação de membros intensa ${ }^{(4)}$. O diagnóstico tardio ou presença de lesões nas artérias aórticas intensas fala a favor de prognóstico reservado ${ }^{(4,11)}$.

O tratamento da SOI depende de como o quadro oftalmológico se encontre. Em pacientes com rubeosis iridis e ângulo aberto, a fotocoagulação pode ser a primeira escolha, não obtendo sucesso pode-se realizar cirurgia filtrante ${ }^{(1)}$. A endarterectomia estabiliza ou obtém alguma melhora visual em $25 \%$ dos pacientes, mas na maioria dos casos falha devido ao dano prévio secundário à hipóxia e glaucoma ${ }^{(11-13)}$.

Diante do quadro da paciente, a nossa conduta foi expectante até termos uma definição do serviço de cirurgia vascular à qual foi encaminhada, para avaliar a possibilidade de tratamento cirúrgico para restaurar o fluxo arterial carotídeo. A importância deste relato é mostrar a necessidade de estarmos conscientes dos problemas sistêmicos que apresentam alterações oftalmológicas. Devemos sempre estabelecer diagnósticos precisos para realizarmos terapêutica adequada, proporcionando uma melhor qualidade e expectativa de vida nesses pacientes.

\section{ABSTRACT}

The ocular ischemic syndrome is a manifestation of chronic hypoperfusion of the ocular arterial supply. Takayasu's arteri- tis is characterized by inflammatory arteritis of big vessels, with variable grades of obstruction, generally aortic branchs. The purpose of this paper is to report a case of ocular ischemic syndrome secondary to Takayasu's arteritis. Digital fluorescein angiography showed signs of chorioretinal hypoperfusion and arteriovenous shunts, and arteriography showed an important reduction of more than $90 \%$ of the blood flow in both right and left carotid arteries.

Keywords: Takayasu's arteritis/complications; Ischemia/etiology; Retinal artery occlusion/diagnosis; Fluorescein angiography/methods; Ocular ischemic syndrome; Retina/physiopathology; Carotid arteries/radiography; Optic nerve/blood supply; Case report

\section{REFERÊNCIAS}

1. Brown GC, Magargal LE, Simeone FA, Goldberg RE, Federman JL, Benson WE. Arterial obstruction and ocular neovascularization. Ophthalmology 1982; 69:139-46.

2. Brown GC, Magargal LE. The ocular ischemic syndrome: clinicalfluorescein angiographic and carotid angiographic features. Int Ophthalmol 1988;11:239-51.

3. Ghirelli W, Amaro MH, Barsante C. Síndrome ocular isquêmica. In: Abujanra S, Ávila MP, Barsante C, Farah M, Gonçalves JOR, Lavinsky J, et al. Retina e vítreo clínica e cirúrgica. São Paulo: Roca; 1999. p.588-91.

4. Haimovici H, Mishima Y. Non atherosclerotic diseases of small arteries. In: Haimovici H. Vascular surgery: principles and techniques. 3th.ed. Norwalk, Conn: Appleton \& Lange; 1989. p.432-5.

5. Inder SJ, Bobryshev YV, Cherian SM, Wang AY, Lord RS, Masuda K, et al. Immunophenotypic analysis of the aortic wall in Takayasu's arteritis: involvement of lymphocytes, dendritic cells and granulocytes in immunoinflamatory reactions. Cardiovasc Surg 2000;8:141-8.

6. Garrido TL, Halfed Neto R, Takahashi WY. Síndrome ocular isquêmica relato de caso. Arq Bras Oftalmol 1998;61:238-41.

7. Raninen RO, Kupari MM, Pamilo MS, Taavitsainen MJ, Poutanen VP, Pajari RI, et al. Ultrassonography in the quantification of arterial involvement in Takayasu's Arteritis. Scand J Rheumatol 2000;29:56-61.

8. Karam EZ, Muci-Mendonza R, Hedges TR. Retinal findings in Takayasu's arteritis. Acta Ophthalmol Scand 1999;77:209-13.

9. Baba T, Itakura K, Tanaka R, Kawasaki T, Kiyosawa M, Numano F. Importance of fluorescein angiographic study in evaluating early retinal changes in Takayasu's disease. Jpn J Ophthalmol 1999;43:546-52.

10. Kiyosawa M, Baba T. Ophthalmological findings in patients with Takayasu's disease. Int J Cardiol 1998;66(Suppl 1):S141-7.; discussion S149.

11. Lambert M, Hachulla E, Hatron PY, Perez-Cousin M, Beregi JP, Warembourg $\mathrm{H}$, et al. Takayasu's arteritis: vascular investigations and therapeutic management. Experience with 16 patients. Rev Med Interne 1998;19:878-84.

12. Johnston ME, Gonder JR, Canny CLB. Successful treatment of ocular ischemic syndrome with panretinal photocoagulation and cerebrovascular surgery. Can J Ophthalmol 1988;123:114-9.

13. Sivalingam A, Brown GC, Magargal LE. The ocular ischemic syndrome. III. Visual prognosis and the effect of treatment. Int Ophthalmol 1991;15:15-20.

\title{
ABO ELETRÔNICO
}

\author{
Novo site
}

\section{Acesso: http://www.abonet.com.br}

